\title{
TOPOLOGICAL RINGS
}

\author{
IRVING KAPLANSKY
}

\section{INTRODUCTION}

1. The literature. Topological rings were apparently first defined in van Dantzig's thesis [13], [14].. Of course innumerable instances of topological rings had been studied earlier: notably Hensel's $p$-adic numbers and their abstraction by Kürschak [54] to fields with valuations. A very considerable literature has since grown up around valuation theory and its applications to algebraic geometry, algebraic functions, and algebraic number theory. Van Dantzig's thesis inaugurated a second stream of investigation: locally compact rings. During the present decade much study has been devoted to Banach algebras (normed rings) in work begun by Gelfand and his colleagues, though foreshadowed by Stone [75], Nagumo [60], and Hebroni [32]. This address is divided into three parts more or less reflecting these three lines of investigation: topological division rings, locally compact rings, and normed algebras.

Besides papers actually referred to in the address, the bibliography includes most of the relevant contributions of the last decade. In certain respects there is no pretense of completeness: for example, there are only a few of the papers on valuations. Particular attention should perhaps be called to the reports of Köthe [52] and Lorch [55], and to the somewhat less closely related reports of Dunford [19], Taylor [78], and Hyers [34].

2. Definitions. By a topological ring we mean a ring and a Hausdorff space in which $a-b$ and $a b$ are jointly continuous in $a$ and $b$. This is substantially the definition of van Dantzig, except that he imposed a countability restriction. A brief account of the fundamentals is presented in Bourbaki [10].

By a normed algebra we mean a normed linear space which is at the same time an algebra satisfying

$$
\|a b\| \leqq\|a\|\|b\| \text {. }
$$

If one merely assumes joint continuity of $a b$, one can pass to an equivalent norm satisfying (1). A Banach algebra is a complete

An address delivered before the Ann Arbor meeting of the Society on April 17, 1948, by invitation of the Committee to Select Hour Speakers for Western Sectional Meetings; received by the editors May 24, 1948.

1 Numbers in brackets refer to the bibliography at the end of the paper. 
normed algebra. It was noted by Gelfand [22, Theorem 1] that in the presence of completeness, separate continuity of $a b$ implies joint continuity. Arens [7, Theorem 5] extended this result to the case of any topological ring whose underlying space is complete metric. In both accounts the proof rests ultimately on a category argument.

To discuss inverses without assuming a unit element we introduce the operation $a \circ b=a+b+a b$, and call $b$ a right quasi-inverse of $a$ if $a \circ b=0$, a quasi-inverse if also $b \circ a=0$. An element is quasiregular if it has a quasi-inverse. Under the operation $\circ$ the quasiregular elements form a group, which will be a topological group if quasi-inversion is continuous. For a normed algebra this continuity can be proved in much the same way as in elementary analysis (cf. $[7$, p. 626]). The continuity of the quasi-inverse is also valid in locally compact rings without divisors of $0[44$, Theorem 8$]$. However, local compactness alone does not suffice, as is shown by an example in $[44]$; nor does it suffice to have a complete metric space $[7, \mathrm{p} .630]$.

To treat maximal ideals in rings without unit it is convenient, following Segal $[69$, p. 74$]$ to introduce regular ideals. A (right, twosided) ideal $I$ is regular if there exists a (left, two-sided) unit modulo $I$. In a Banach algebra all regular maximal ideals are closed. More generally this is true in any ring in which the right quasi-regular elements form an open set; such rings are called $Q_{r}$-rings in [40]. If the quasi-regular elements are open (a formally stronger condition), the ring is called a $Q$-ring.

We shall use the Perlis-Jacobson radical, defined in [38] to be the union of the right quasi-regular ideals; it may be shown to be the intersection of the right (or left) regular maximal ideals. Hence in a $Q_{r}$-ring the radical is closed. The radical is also closed if the right quasi-regular elements form a closed set, which is true for example in a compact ring. But the radical is not always closed, as is shown by an example in [40]. In certain respects the following is a simpler example. Let $P$ be the ring of $p$-adic integers and $A$ the ring of all infinite matrices over $P$, with only a finite number of nonzero elements in each row. We topologize $A$ with the "finite" topology: the general neighborhood of 0 consists of all matrices with first $n$ rows 0 . (The $p$-adic topology in $P$ is ignored; in fact any ring with a non-nil radical will do in place of $P$.) Then it is easily checked that the radical $R$ of $A$ contains all matrices with only a finite number of nonzero entries, and these all divisible by $p$. The matrix $p\left(e_{12}+e_{23}+e_{34}+\cdots\right)$ therefore lies in the closure of $R$, but it is not itself in $R$, since it is not even quasi-regular.

A useful concept in the study of topological rings is that of bound- 
edness, first introduced by Shafarevich [72], and presumably inspired by the analogous concept for topological linear spaces. A set $S$ in a topological ring is right bounded if for any neighborhood $U$ of 0 there exists a neighborhood $V$ of 0 such that $S V \subset U$. Left boundedness is analogously defined, and a set is bounded if it is both right and left bounded. Compact sets are bounded, and for algebraic purposes bounded sets may often be regarded as the appropriate generalization of compact sets. For example, a polynomial is uniformly continuous on any bounded set; and if the quasi-inverse is continuous, then it is uniformly continuous on any bounded set.

\section{Topological DIVISION RINGS}

3. Normed division algebras. Mazur announced in [58] the theorem that the only (complex) normed division algebra is the field of complex numbers itself. Gelfand proved this in [22], asserting that his proof was different from Mazur's. Gelfand's proof rests on an application of Liouville's theorem and, as Lorch has remarked, substantially the same result had been given earlier by Taylor [77]; the same sort of argument occurs in many older references.

We present this Taylor-Gelfand argument in a somewhat generalized form substantially due to Arens [7]. Let $A$ be a topological algebra over the complex numbers. We assume that $A$ has a unit, that the inverse in $A$ is continuous, and that $A$ admits a total set of functionals (the latter is true for example if $A$, as a topological linear space, is convex). The spectrum of $x$ is the set of scalars $\lambda$ such that $(x-\lambda)^{-1}$ does not exist. We assert that every element of $A$ has a nonvoid spectrum. For if not, we form for an arbitrary functional $f$ the function $g(\lambda)=f\left[(x-\lambda)^{-1}\right]$, and verify that $g(\lambda)$ is entire and approaches 0 as $\lambda \rightarrow \infty$. By Liouville's theorem, $g \equiv 0$, whence $(x-\lambda)^{-1}$ $=0$, a contradiction. If we specialize $A$ to be a division algebra, we deduce the Gelfand-Mazur theorem: every element of $A$ is a scalar multiple of the unit. By a standard embedding device one obtains a corresponding theorem in the real case; here the reals, complexes or quaternions may arise.

While the above argument appears to have squeezed everything possible out of Liouville's theorem, it seems probable that stronger theorems are true, and perhaps even that no nontrivial topological division algebras over the complexes exist at all. The question may be rephrased thus: is it possible to convert the field of rational functions $f(z)$ of a complex variable $z$ into a topological algebra? An easily proved result in this connection is that it cannot be done if $f(z)$ is to be continuous jointly in $f$ and $z$. 
Returning to normed algebras, we mention two generalizations of the Gelfand-Mazur theorem which are given in [46] and [45] respectively. (1) Call $x$ a topological divisor of 0 if there exists a non-null sequence $y_{n}$ such that $x y_{n}$ or $y_{n} x \rightarrow 0$. The following theorem is a refinement of results in [73], [7] and [68]: a normed algebra without any nonzero topological divisors of 0 is the reals, complexes, or quaternions. It is to be observed that neither a unit element nor completeness is assumed. (2) A Banach algebra which is regular in the sense of von Neumann (for any $a$ there exists an $x$ such that $a x a=a$ ) is finite-dimensional. Here completeness is indispensable.

Topological linear spaces are of course necessarily connected, and it is perhaps natural, in connection with the above results, to ask what connected fields exist. In a somewhat different context, much the same question was raised by Baer and Hasse [9]. Some light has been thrown on this question recently, by Dieudonné [17] who constructed a connected subfield of the complex numbers, and Kapuano [47] who constructed a one-dimensional subfield of the complex numbers (in both cases a field other than the reals, of course).

4. Valuations. Kürschak [54] defined a valuation of a division ring to be a real-valued function satisfying $|a| \geqq 0,|a|=0$ if and only if $a=0,|a b|=|a||b|,|a+b| \leqq|a|+|b|$. He was inspired by two outstanding examples: complex numbers and $p$-adic numbers. Ostrowski [61] showed that these two examples are typical. A field with a valuation is either (archimedean case) a subfield of the complex numbers with essentially its ordinary absolute value, or (nonarchimedean case) it satisfies with the $p$-adic numbers the strong triangle inequalty

$$
|a+b| \leqq \max (|a|,|b|) \text {. }
$$

Thus structure theory is of interest only in the non-archimedean case, although in applications (notably algebraic number theory) it remains important to consider all valuations. Krull [53] took the next step of observing that in (2), addition of real numbers has disappeared; only the multiplicative group of positive real numbers is relevant, and one may generalize by replacing it by an arbitrary ordered abelian group $\Gamma$. The classification of fields with valuations was successfully studied in case $\Gamma$ is the additive group of integers; $c$. Teichmüller [79] and MacLane [56]. For an arbitrary group $\Gamma$, definite but more complicated results were obtained in [39]. A similar study has been made for local rings by Cohen [12].

Examination of some of the proofs in valuation theory reveals that full use is not made of the existence of a valuation. In many cases 
one can get by with a topological field having the following property: if a set $S$ is bounded away from 0 , then $S^{-1}$ is bounded (in the sense of \$2). In [41], division rings with this property are said to be of "type V." The same class of division rings is briefly studied in an exercise in Bourbaki [10, p. 57, Ex. 13].

The following are examples of theorems which carry over to the case of type V. (1) A finite-dimensional topological linear space over a complete division ring of type $\mathrm{V}$ necessarily carries the Cartesian product topology. (2) The completion of an algebraically closed field of type $\mathrm{V}$ is algebraically closed. It has yet to be determined whether a finite algebraic extension of a field of type $\mathrm{V}$ can be topologized so as to be of type $\mathrm{V}$. This is proved for quadratic extensions in [41].

Fields with an (ordinary real-valued) valuation can be characterized as being of type $\mathrm{V}$ and having a neighborhood of 0 consisting of topologically nilpotent elements [41, Theorem 3]. Zelinsky [83] has similarly characterized fields with a non-archimedean valuation. As a test problem for further progress, one may propose the following question: how can the field of rational numbers be topologized so as to be of type V? Are there any such topologies other than those given by valuations?

We mention finally [42], in which there is presented a topological discussion of polynomials in fields of type $\mathrm{V}$. In particular a recent theorem of Habicht [31] on real closed fields is generalized. (It is to be observed that any ordered field in its order topology is of type V.)

5. Locally compact division rings. With the examples of the real numbers and $p$-acid numbers before one, it is natural to ask what locally compact fields exist, and in particular if any exist essentially different from these two examples. Such an investigation was undertaken independently by van Dantzig [13] and Pontrjagin [64]. The latter showed that the only locally compact connected division rings are the reals, complexes, and quaternions. (In his book [65, pp. 171178 ] he assumes the second axiom of countability, but no countability assumption is made in the paper.) Van Dantzig confined himself to the commutative case and assumed the second axiom of countability. In the connected case he obtained the result cited above (excluding of course the quaternions). He also treated the totally disconnected case, obtaining either a finite extension of the $p$-adic numbers (a " $\pi$-adic field") or the field of formal power series over a finite field (a "z-adic field"). Jacobson [36] completed the problem by treating the non-commutative totally disconnected case, showing that one gets an algebra of finite order over a $p$-adic or $z$-adic field; known struc- 
ture theory shows that the algebra is cyclic. Jacobson assumed the first axiom of countability in his proof; however the opening lines in Pontrjagin's paper constitute a proof that any locally compact division ring satisfies the first axiom of countability. Otobe [62] proved again this superfluity of a countability assumption. It may be remarked that slightly later in the argument one of course gets the second axiom of countability free of charge (always assuming, as was tacitly done above, that the topology is not discrete).

Thus the structure of locally compact division rings was fully determined. The elegance of the result has attracted further study since then. Shafarevich [72] made the following contribution: he gave a characterization of those topological fields which admit a valuation giving the same topology. The conditions in question are readily verifiable in a locally compact field, and so the problem is reduced to one in valuation theory. The known theorems on valuations readily complete the solution. In the non-commutative case there are some additional difficulties which were overcome in [41]. A noteworthy feature of this solution of the problem is that it treats the connected and totally disconnected cases on precisely the same footing.

The latter merit is also shared by the interesting proof announced by Braconnier [11]. He observes that the mapping $a \rightarrow a x(x \neq 0)$ is a bicontinuous automorphism of the additive group of a locally compact division ring. The uniqueness of Haar measure shows that this mapping multiplies all measures by a positive real number $v(x)$. One then verifies that $v(x)$ is in fact a valuation. From this point on, valuation theory is presumably to be applied.

Various generalizations of the Pontrjagin-van Dantzig-Jacobson theory might be proposed. Locally compact rings which are not necessarily division rings will be discussed in Part III of this address. The hypothesis of local compactness might be weakened, perhaps most plausibly to local boundedness. Finally we shall mention the weakening or dropping of the associative law. This has some interest from the point of view of the foundation of geometry-cf. Kolmogoroff [50] and Köthe [52]; and, in view of the work of Ruth Moufang, particular interest is attached to alternative division rings. The connected case falls under the results discussed in $\$ 6$ below. It appears that nothing is known in the totally disconnected case; but one may venture the conjecture that the only totally disconnected locally compact alternative division rings are Cayley-Dickson algebras over a $p$-adic or $z$-adic field. The methods that work in the associative case do not seem to apply without very considerable modification, though perhaps Braconnier's is the most hopeful. 


\section{LOCALLY COMPACT RINGS}

6. The component of 0 . A great deal is known about the structure of locally compact abelian groups, mainly through the work of Pontrjagin and van Kampen. Expositions can be found in [65] and [80]. Thus the study of locally compact rings begins with the observation that the structure of the underlying additive group is substantially known. But even if the additive group is completely known, this does not mean that there is nothing left to study in the ring. To cite an extreme case: finite abelian groups are completely known, but it can hardly be said that there are no unsolved questions about finite rings.

Nevertheless the additive theory has provided strong tools for ring investigations. This is particularly notable in the connected case, where fairly decisive results were obtained by Jacobson and Taussky [35]. The heart of the argument is given in [44] in the following general form: let $A$ be a locally compact ring, $C$ the component of 0 , and $B$ a right bounded additive subgroup of $A$; then $C B=0$. This is a fairly immediate consequence of the existence of sufficiently many characters of the additive group of $A$, which in turn rests ultimately on the Peter-Weyl theorem. Indeed the cited result is true under the mere hypothesis (in lieu of local compactness) of the existence of sufficiently many characters, or even the still weaker hypothesis that there exist sufficiently many continuous homomorphisms into a group with no arbitrarily small subgroups.

We quote next the structure theorem which asserts that $A$ is the direct sum of a vector group $N$ and a group in which the component $P$ of 0 is compact $\left[80\right.$, p. 110], and we deduce $P^{2}=P N=N P=0$. In particular if $A$ is compact and connected, $A^{2}=0$. In any event, if $A$ is connected, the hypothesis that there is no total divisor of 0 will entail $P=0, A=N$, and it follows readily that $A$ is an algebra of finite order over the reals. If it is an associative division algebra we get the theorem of Pontrjagin, and if alternative, $A$ may further be the Cayley numbers. We may note at this point that these four algebras have also been characterized by Albert [1] as being precisely those that admit valuations which are homogeneous with respect to real scalars.

Something further can be said about locally compact connected rings without the assumption that there are no total divisors of 0 . We in any event have the additive decomposition $A=N+P$, with $P A=A P=0$. We may thus describe the structure of $A$ as follows: $N$ is an algebra of finite order over the reals, $P$ a compact abelian group, and multiplication takes place in $A$ according to the rule 


$$
\left(n_{1}+p_{1}\right)\left(n_{2}+p_{2}\right)=n_{1} n_{2}+f\left(n_{1}, n_{2}\right)
$$

where $n_{i} \in N, p_{i} \in P$, and $f$ is a continuous homomorphism of the Kronecker product $N \times N$ into $P$. To some extent one can prescribe $f$ more fully in terms of the structure of $N$, but we shall not enter into details. The following remark may however be of interest. If $A$ is locally connected, then $P$ is a direct sum of circle groups, and $f$ is a direct sum of characters. If we write $\left\{u_{i}\right\}$ for a basis of $N$, then the characters in question take the form

$$
\left(\sum x_{i} u_{i}, \sum y_{j} u_{j}\right) \rightarrow \sum t_{i j} x_{i} y_{j}(\bmod 1),
$$

$t_{i j}$ being real numbers. If finally $A$ is locally Euclidean, this remark shows that a local coordinate system is introducible in which ring multiplication is a bilinear function. The analogue of Hilbert's fifth problem for rings thus has a strongly affirmative answer.

If we are given an arbitrary locally compact ring $A$, then the preceding discussion may be applied to its component $C$ of 0 . To some extent, questions on the structure of $A$ may be reduced to the analogous ones for the connected ring $C$ and the totally disconnected ring $A-C$; details are given in [44]. In the remainder of our discussion of locally compact rings we shall confine ourselves to the totally disconnected case.

7. The compact case. The fundamental feature of the totally disconnected case is the existence of group neighborhoods of 0 , a result due to van Kampen. For a compact ring $A$ we may pass at once to ideal neighborhoods of 0 ; if $U$ is a group neighborhood we can find $V$ such that the open ideal $V+A V+V A+A V A$ is contained in $U$. Thus a compact totally disconnected ring is $b_{\nu}$-adic in the sense of van Dantzig [15]. This fact makes possible a workable theory of the radical and semi-simplicity, as presented in [40].

Call an ideal $I$ topologically nilpotent if for any neighborhood $U$ of 0 there exists an integer $n$ such that $I^{n} \subset U$. We begin with the theorem that the radical of $A$ is topologically nilpotent; this is an immediate consequence of the ideal neighborhoods and the nilpotence of the radical of a finite ring. We can then proceed to a complete determination of compact semi-simple rings. Theorem: they are Cartesian direct sums of (any number of) finite simple rings. We shall sketch a proof which is slightly simpler than that in [40], the simplification resting on the use of regular ideals. First we show that in a compact semi-simple ring $A$ there exist open regular maximal ideals, and that their intersection is 0 ; the proof in [40], for the cor- 
responding statement with the word "regular" deleted, is valid almost unchanged. This already proves that $A$ is a subdirect sum of finite simple rings. The structure theory of finite rings shows moreover that any finite combination of coordinates occurs; because of the compactness we readily pass to the complete direct sum.

This determination of compact semi-simple rings makes possible some results on the non-semi-simple case. These are obtained by the classical procedure of constructing in $A$ idempotents which map on the idempotents of $A-R$, where $R$ is the radical. It is appropriate to cite at this point the following unpublished remark due to Jacobson. The construction of idempotents in question can be carried through in a ring $A$ if its radical $R$ satisfies the following condition: for any $y \in R$ the equation $x^{2}+x=y$ can be solved for $x$ in $R$. The solvability of this equation can be established by a series expansion which is valid if $y$ is nilpotent in the ordinary sense. In our topological context, nilpotence of $y$ in the topological sense will do, provided there is some further assurance of the convergence of the series. For compact rings, this assurance is provided by the group neighborhoods; in Banach algebras the convergence can be established by estimations of the norm. It may be well to remark that there exist rings where $x^{2}+x=y$ is not always solvable in the radical-an example is furnished by the ring of all rational numbers with odd denominators. In a sense we may ascribe the failure of this ring to the fact that it is not complete in its natural (2-adic) topology.

The ability to construct idempotents carries with it certain structure theorems for compact rings, notably an additive decomposition into primary rings, and the fact that a primary ring is the ring of matrices over a completely primary ring. Besides the light they shed on compact rings, these results are useful in the locally compact case as we shall see in $\S 8$.

This comparatively complete array of structure theorems runs of course parallel to the classical theory of rings with descending chain condition. It is natural to ask whether one can formulate a theory covering both cases at once. In [44] it is shown that a suitable set of axioms for this purpose is given by: (1) local compactness, (2) boundedness, (3) the descending chain condition for right ideals containing a fixed open two-sided ideal.

8. Compact subrings. Let $A$ be a locally compact totally disconnected ring. We know that $A$ has a system of group neighborhoods of 0 ; but we cannot pass to ideal neighborhoods of 0 as we did in the compact case. However we can achieve the next best thing: subring 
neighborhoods of 0 . The argument for this is implicit in the work of Jacobson [36] and runs as follows. For a compact open subgroup $U$ select an open subgroup $V$ such that $V \subset U, V U \subset U$. Then $W=V$ $+V^{2}+V^{3}+\cdots$ is a compact open subring contained in $U$.

The existence of these compact open subrings provides a foothold from which an invasion of locally compact rings becomes possible to some extent, and is launched in [44]. The spirit of the investigation is as follows. Let $B$ be a compact open subring of $A$. We subdivide into two cases. (1) The radical of $B$ is open: in this case $B$, and hence also $A$, is a $Q$-ring. (2) The radical of $B$ is not open: in this case there exist idempotents arbitrarily near zero, by the compact ring theory. In either eventuality we have something from which we may hope to proceed further.

We mention two theorems which are proved in this way in [44]. (a) If $A$ has no divisors of 0 , or under any of several alternative hypotheses, we can rule out case (2) and conclude unreservedly that $A$ is a $Q$-ring. (b) If $A$ is not a radical ring it contains at least one closed regular maximal right ideal. Some contributions are also made in [44] to the structure theory of simple and semi-simple locally compact rings, but a reasonably complete theory has yet to be constructed.

\section{NORMED ALGEBRAS}

9. The radical. The fundamental result on the radical of a Banach algebra is due to Gelfand [22]; cf. also Jacobson [38]. We present a somewhat generalized version. Let $A$ be a complex Banach algebra. As is appropriate if $A$ does not have a unit, we define the spectrum of $x \in A$ to consist of all scalars $\lambda$ such that $-\lambda^{-1} x$ does not have a quasi-inverse. Then

$$
\sup |\operatorname{spectrum}(x)|=\lim _{n \rightarrow \infty}\left\|x^{n}\right\|^{1 / n} .
$$

The proof rests on the expansion of the quasi-inverse of $-\lambda^{-1} x$ as a power series in $\lambda$ and examination of the radius of convergence; by taking functionals one can reduce the problem to the case of ordinary analytic functions. With a suitable definition of the spectrum, (3) can also be extended to a real Banach algebra.

If in particular $x$ is in the radical, then its spectrum contains only 0 , and it follows that $x^{n} \rightarrow 0$. One easily deduces that the radical is the union of all nil ideals, a nil ideal being one consisting entirely of topologically nilpotent elements. It seems remarkable that this nilpotence of the elements of the radical is valid both for compact rings 
and Banach algebras, but must be established by such different methods.

10. Semi-simple algebras. The analogy between Banach algebras and rings with a finiteness condition persists to some extent in the structure theory of semi-simple algebras; that is, in certain cases we obtain a (suitably generalized) direct sum of (suitably generalized) matrix algebras. Of course in the commutative case the matrix algebras collapse. We mention at this point that great simplifications also occur in the case of completely continuous Banach algebras (that is, algebras in which the mappings $x \rightarrow a x$ and $x \rightarrow x a$ are completely continuous). Such algebras are studied in [21] and [46].

Probably the most satisfactory structure theorem for commutative Banach algebras is due to Gelfand and Neumark [26]. Let $A$ be a complex commutative Banach algebra with unit, and suppose there is an involution $x \rightarrow x^{*}$ which is conjugate linear and satisfies $\left\|x x^{*}\right\|$ $=\|x\|\left\|x^{*}\right\|$. Then $A$ is isomorphic to the algebra of all continuous complex functions on a compact Hausdorff space, with the norm identified as sup, and the * identified as conjugate. An obscure point in the Gelfand-Neumark proof was cleared up by Arens [5], who also [6] extended the theorem to the case where no unit is assumed. Analogous characterizations of the algebra of all real functions are given in [6] and [71]; and in [8] the real and complex cases are united.

There are important applications of such a characterization of the ring of continuous functions. We mention two in particular: the Stone-Cech compactification of a completely regular space, and the spectral theorem for a bounded normal operator on Hilbert space. Various accounts of these two applications can be found in the recent literature. Another interesting example on which to test the power of such a characterization is the theorem proved by Stone in [75] and Eidelheit in [20]: a closed sub-algebra of the algebra of continuous functions on a compact Hausdorff space is itself an algebra of all continuous functions on a compact Hausdorff space.

The main theorem of Gelfand and Neumark in [26] concerned noncommutative Banach algebras. To the axioms listed above one adds: $\left\|x^{*}\right\|=\|x\|$ and $1+x x^{*}$ has an inverse (it is not known whether these two axioms are independent of the preceding ones). It is then shown that the algebra is isomorphic to a closed self-adjoint subalgebra of the algebra of all bounded operators on a Hilbert space. This is an important and elegant theorem, but it should be pointed out that the result merely identifies the algebra as being a subset of something, and for many purposes this is a serious drawback. 
11. $H^{*}$-algebras. There is a structure theorem for noncommutative Banach algebras which does effect a complete identification of the algebras in question as being a full direct sum of full matrix algebras, in remarkable analogy to the finite-dimensional case. The theorem applies to the $H^{*}$-algebras of Ambrose [2]. The axioms are as follows: $A$ is a Hilbert space and a Banach algebra; there is an involution which is conjugate linear and satisfies $(x y, z)=\left(y, x^{*} z\right),(y x, z)$ $=\left(y, z x^{*}\right)$ where the parentheses denote the Hilbert space inner product; and finally we add an axiom assuring nontriviality: $x A=0$ implies $x=0$. The structure theorem asser ts that $A$ is a direct sum of simple $H^{*}$-algebras, the direct sum being meant in the Hilbert space sense; and a simple $H^{*}$-algebra is the set of all matrices $a=\left(a_{i j}\right)$ of complex numbers with $\sum\left|a_{i j}\right|^{2}<\infty$ under ordinary matrix multiplication, with

$$
(a, b)=\alpha \sum a_{i j} b_{i j}^{c}
$$

and $a^{*}=\left(a_{j_{i}}\right)^{c}$ (the superscript denoting complex conjugate). In [43] a somewhat simplified proof is given, and the theorem is extended to real $H^{*}$-algebras.

The outstanding application of this theorem is to the $L_{2}$-algebra of a compact group. Let $G$ be a compact group and $A$ the algebra of complex functions square-summable with respect to the Haar measure of $G$. We use the $L_{2}$-norm, define multiplication by convolution

$$
f g(x)=\int f(y) g\left(y^{-1} x\right) d y,
$$

and set $f^{*}(x)=f\left(x^{-1}\right)^{\text {c }}$. Then it follows directly from elementary properties of Haar measure and convolution that $A$ is an $H^{*}$-algebra, and so the above structure theorem is applicable. It should be remarked that this highly algebraic approach to the study of compact groups was initiated by Köthe [51]; however, he based his work on the algebra of continuous functions instead of on $L_{2}$, and his results are not quite as satisfactory as those of Ambrose.

The fact that $A$ is an $H^{*}$-algebra contains the Peter-Weyl theorem and related facts. In order to see this, one has to show (among other things) that these particular $H^{*}$-algebras have simple components that are finite-dimensional. As indicated by Ambrose, this can be done by appealing to the fact that $A$ is a completely continuous algebra. However it is possible to deduce the finite-dimensionality without using any further information from the group, except for some rudimentary integration, and thus we can arrive at the Peter-Weyl theorem without mentioning the words "completely continuous." 
The computation at the same time establishes the connection between the algebra $A$ and the representations of $G$, the orthogonality relations, and so on.

One proceeds as follows. Let $e_{i j}$ denote matric units for one of the simple components of $A$. In (5) set $f=e_{i k}, g=e_{k j}, x=1=$ the group identity. Then

$$
e_{i j}(1)=\int e_{i k}(y) e_{k j}\left(y^{-1}\right) d y=\left(e_{i k}, e_{j k}\right)=\alpha \delta_{i j},
$$

the last equality following from (4). Next define $h$ by $h(x)=e_{i j}(x y)$; it is easy to see that $h$ is in the right ideal generated by $e_{i j}$, say $h=\sum_{k} a_{k} e_{i k}$. On setting $x=1$ we obtain $e_{i j}(y)=\alpha \sum a_{k} \delta_{i k}=\alpha a_{i}$. Hence

$$
e_{i j}(x y)=\alpha^{-1} \sum_{k} e_{i k}(x) e_{k j}(y) .
$$

Set $y=x^{-1}, i=j=1$ in (6) and integrate over the group (the total measure is assumed to be unity).

$$
\alpha=\alpha^{-1} \sum_{k}\left(e_{1 k}, e_{1 k}\right) .
$$

From (7) it follows that the sum on $k$ must be finite, and hence that the matrix algebra must be finite-dimensional; in fact $\alpha$ is precisely the degree of the matrices. Equation (6) shows that $x \rightarrow \alpha^{-1} e_{i j}(x)$ is a unitary representation of $G$, which one proves to be irreducible by standard methods. This substantially establishes the Peter-Weyl theorem and its attendant facts.

12. Group algebras. Let $G$ be a locally compact group and $A$ the $L_{1}$-algebra of $G$, that is, the algebra of all complex functions summable with respect to the left Haar measure of $G$, with convolution as multiplication. The investigation of these Banach algebras is a subject of considerable current interest, which promises to produce results of first-rate importance.

It is appropriate to begin the discussion with the theorem that $A$ is semi-simple. Segal [69] proves this neatly as follows. He remarks that any self-adjoint algebra of bounded operators on Hilbert space is semi-simple; this is a direct corollary of the spectral theorem, or rather of that fragment of it which asserts that a bounded self-adjoint operator with only 0 in its spectrum is necessarily 0 . The result then follows from the fact that $A$ is faithfully represented by operators on $L_{2}(G)$. In certain cases Segal proves that the intersection of the regular maximal two-sided ideals is 0 ; this is a statement which is 
stronger than semi-simplicity. Raikov [66] has given a different proof of the semi-simplicity of $A$.

This semi-simplicity can be used to extablish the existence of a complete set of irreducible representations of $G$ by operators on Banach spaces, called $B$-representations in [69]. If $G$ is compact or abelian, these $B$-representations are finite-dimensional and hence similar to unitary representations. In the general case it is not known whether the $B$-representations are similar to unitary representations on Hilbert space; lack of information on this point is one of the obstacles impeding further progress on the structure of the $L_{1}$-algebra. It has been proved [28], [70] that $G$ has a complete set of irreducible unitary representations. In the latter reference the proof is carried out by regarding $A$ as an algebra of operators on $L_{2}(G)$ and taking the uniform closure.

We turn now to the case where $A$ is commutative (which is true if and only if $G$ is), and we shall briefly discuss ideal theory in $A$. Let $I \neq A$ be a closed ideal in $A$. We first raise the question: is $I$ contained in a regular maximal ideal? This is immediate if $A$ has a unit-which is true if and only if $G$ is discrete. This easy result, plus the information that the maximal ideals correspond precisely to the characters of $G$, constitutes a generalization of Wiener's theorem on the reciprocal of an absolutely convergent trigonometric series. Even if $A$ does not have a unit, it is still true that $I$ can be embedded in a regular maximal ideal, although it requires a fair amount of proof-cf. [69] and [30]. It should be noted that the standard process of adjoining a unit does not resolve the difficulty, and in fact it is technically preferable not to adjoin a unit. In the case where $G$ is the group of real numbers, this is the theorem of Wiener on spanning $A$ by translates of a function whose Fourier transform does not vanish.

The question just raised might have been paraphrased as follows: is $A-I$ a radical ring (for only then would it be impossible to embed $I$ in a regular maximal ideal)? A more far-reaching question suggests itself: is $A-I$ even semi-simple? Even for specific groups like the integers or reals, this is still an open question. For special choices of the ideal $I$, it has been answered affirmatively in [18] and [69], and these partial results are generalizations of Tauberian theorems of Wiener and Pitt.

Such is the state of our knowledge in the commutative case. In the noncommutative case there are as yet few results concerning $L_{1}(G)$ worth quoting. An attack in a different direction has been made by Ambrose [3] who proposes to study $L_{2}(G)$. This is not an algebra since it is not closed under convolution, but in compensation for this 
difficulty one has the availability of Hilbert space techniques. A somewhat similar program has been announced by Mautner [57]. Under the assumption that $G$ is separable, he decomposes unitary representations of $G$, and in particular the regular representation, obtaining an analogue of Plancherel's theorem. The decomposition is based on a theorem of von Neumann concerning the expression of a ring of operators as a direct integral of irreducible rings.

\section{BIBLIOGRAPHY}

1. A. A. Albert, Absolute valued real algebras, Ann. of Math. vol. 48 (1947) pp. 495-501.

2. W. Ambrose, Structure theorems for a special class of Banach algebras, Trans. Amer. Math. Soc. vol. 57 (1945) pp. 364-386.

3. - The $L_{2}$-system of a unimodular group, To be published in Trans. Amer. Math. Soc.

4. H. Anzai, On compact topological rings, Proc. Imp. Acad. Tokyo vol. 19 (1943) pp. 613-615.

5. R. Arens, On a theorem of Gelfand and Neumark, Proc. Nat. Acad. Sci. U. S. A. vol. 32 (1946) pp. 237-239.

6. - , Representation of *-algebras, Duke Math. J. vol. 14 (1947) pp. 269-282.

7. - Linear topological division algebras, Bull. Amer. Math. Soc. vol. 53 (1947) pp. 623-630.

8. R. Arens and I. Kaplansky. Topological representation of algebras, Trans. Amer. Math. Soc. vol. 63 (1948) pp. 457-481.

9. R. Baer and H. Hasse, Zusammenhang und Dimension topologischer Körperraume, Journal für Mathematik vol. 167 (1932) pp. 40-45.

10. N. Bourbaki, Eléments de mathématique, vol. 3, Topologie générale, Actualités Scientifiques et Industrielles, no. 916, Paris, 1942.

11. J. Braconnier, Groupes d'automorphismes d'un groupe localement compact, C. R. Acad. Sci. Paris vol. 220 (1945) pp. 382-384.

12. I. S. Cohen, On the structure and ideal theory of complete local rings, Trans. Amer. Math. Soc. vol. 59 (1946) pp. 54-106.

13. D. van Dantzig, Studiën over topologische algebra, Dissertation, Amsterdam, H. J. Paris, 1931.

14. - Zur topologische Algebra, Math. Ann. vol. 107 (1933) pp. 587-626.

15. — Zur topologische Algebra II, Compositio Math. vol. 2 (1935) pp. 201223.

16. - Neuere Ergebnisse der topologischen Algebra, Rec. Math. (Mat. Sbornik) N.S. vol. 1 (1936) pp. 665-674.

17. J. Dieudonne, Sur les corps topologiques connexes, C. R. Acad. Sci. Paris vol. 221 (1945) pp. 396-398.

18. V. Ditkin, On the structure of ideals in certain normed rings, Uchenye Zapiski. Moskov. Gos. Univ. Mat. vol. 30 (1939) pp. 83-130. 651.

19. N. Dunford, Spectral theory, Bull. Amer. Math. Soc. vol. 49 (1943) pp. 637-

20. M. Eidelheit, Concerning rings of continuous functions, Ann. of Math. vol. 41 (1940) pp. 391-393.

21. M. Freundlich, On normed rings, Dissertation, Illinois, 1947. 
22. I. Gelfand, Normierte Ringe, Rec. Math. (Mat. Sbornik) N.S. vol. 9 (1941) pp. 3-24.

23. - Ideale und primäre Ideale in normierten Ringen, Rec. Math. (Mat. Sbornik) N.S. vol. 9 (1941) pp. 41-48.

24. - Zur Theorie der Charaktere der abelschen topologischen Gruppen, Rec. Math. (Mat. Sbornik) N.S. vol. 9 (1941) pp. 49-50.

25. - Über absolut konvergente trigonometrische Reihen und Integrale, Rec. Math. (Mat. Sbornik) N.S. vol. 9 (1941) pp. 51-66.

26. I. Gelfand and $M$. Neumark, On the imbedding of normed rings into the ring of operators in Hilbert space, Rec. Math. (Mat. Sbornik) N.S. vol. 12 (1943) pp. 197-213.

27. I. Gelfand and D. Raikov, On the theory of characters of commutative topological groups, C. R. (Doklady) Acad. Sci. URSS. vol. 28 (1940) pp. 195-198.

28. - Irreducible unitary representations of locally compact groups, Rec. Math. (Mat. Sbornik) N.S. vol. 13 (1943) pp. 301-316.

29. R. Godement, Les fonctions de type positif et la thêorie des groupes, Trans. Amer. Math. Soc. vol. 63 (1948) pp. 1-84.

30. — Theorèmes tauberiens et theorie spectrale, Ann. Ecole Norm. vol. 74 (1947) pp. 119-138.

31. W. Habicht, Ein Existenzsatz ibber reele definite Polynome, Comment. Math. Helv. vol. 18 (1946) pp. 331-348.

32. P. Hebroni, Über lineare Differentialgleichungen in Ringen.$\cdots$, Compositio Math. vol. 5 (1938) pp. 403-429.

33. E. Hewitt, On rings of continuous real-valued functions, Trans. Amer. Math. Soc. vol. 64 (1948) pp. 45-99.

34. D. Hyers, Linear topological spaces, Bull. Amer. Math. Soc. vol. 51 (1945) pp. $1-21$.

35. N. Jacobson and O. Taussky, Locally compact rings, Proc. Nat. Acad. Sci. U. S. A. vol. 21 (1935) pp. 106-108.

36. N. Jacobson, Totally disconnected locally compact rings, Amer. J. Math. vol. 58 (1936) pp. 433-449.

37. - A note on topological fields, Amer. J. Math. vol. 59 (1937) pp. 889-894.

38. - The radical and semi-simplicity for arbitrary rings, Amer. J. Math. vol. 67 (1945) pp. 300-320.

39. I. Kaplansky, Maximal fields with valuations, Duke Math. J. vol. 9 (1942) pp. 303-321, vol. 12 (1945) pp. 243-248.

40. - Topological rings, Amer. J. Math. vol. 69 (1947) pp. 153-183.

41. —-, Topological methods in valuation theory, Duke Math. J. vol. 14 (1947) pp. 527-541. Soc.

42. — Polynomials in topological fields, To be published in Bull. Amer. Math.

43. - Dual rings, Ann. of Math. vol. 49 (1948) pp. 689-701.

44. - Locally compact rings, Amer. J. Math. vol. 70 (1948) pp. 447-459.

45. - Regular Banach algebras, To appear in J. Indian Math. Soc.

46. - Normed algebras, in preparation.

47. I. Kapuano, Sur les corps de nombres a une dimension distincts du corps réel, Rev. Fac. Sci. Univ. Istanbul (A) vol. 11 (1946) pp. 30-39.

48. Y. Kawada, Über die Erweiterung der maximalen Ideale in normierten Ringen, Proc. Imp. Acad. Tokyo vol. 19 (1943) pp. 267-268.

49. K. Kodaira and S. Kakutani, Normed ring of a locally compact abelian group, Proc. Imp. Acad. Tokyo vol. 19 (1943) pp. 360-365. 
50. A. Kolmogoroff, Zur Begrilndung der projektiven Geometrie, Ann. of Math. vol. 33 (1932) pp. 175-176.

51. G. Köthe, Abstrakte Theorie nichtkommutativer Ringe mit einer Anwendung auf die Darstellungstheorie kontinuierlicher Gruppen, Math. Ann. vol. 103 (1930) pp. 545572.

52. - Unendliche abelsche Gruppen und Grundlagen der Geometrie, Jber. Deutschen Math. Verein. vol. 49 (1939) pp. 97-113.

53. W. Krull, Allgemeine Bewertungstheorie, Journal für Mathematik vol. 167 (1932) pp. 160-196.

54. J. Kürschak, Über Limesbildung und allgemeine Körpertheorie, Journal für Mathematik vol. 142 (1913) pp. 211-253.

55. E. Lorch, The structure of normed abelian rings, Bull. Amer. Math. Soc. vol. 50 (1944) pp. 447-463.

56. S. MacLane, Subfields and automorphism groups of p-adic fields, Ann. of Math. vol. 40 (1939) pp. 423-442.

57. F. Mautner, The completeness of the irreducible unitary representations of a locally compact group, Proc. Nat. Acad. Sci. U. S. A. vol. 34 (1948) pp. 52-54.

58. S. Mazur, Sur les anneaux linéaires, C. R. Acad. Sci. Paris vol. 207 (1938) pp. $1025-1027$.

59. D. Milman, On the normalizability of topological rings, C. R. (Doklady) Acad. Sci. URSS. vol. 47 (1945) pp. 162-164.

60. M. Nagumo, Einige analytische Untersuchungen in linearen, metrischen Ringen, Jap. J. Math. vol. 13 (1936) pp. 61-80.

61. A. Ostrowski, Über einige Lösungen der Funktionalgleichung $\phi(x) \phi(y)=\phi(x y)$, Acta Math. vol. 41 (1918) pp. 271-284.

62. Y. Otobe, On locally compact fields, Jap. J. Math. vol. 19 (1945) pp. 189-202.

63. F. Peter and H. Weyl, Die Vollständigkeit der primitiven Darstellungen einer geschlossenen kontinuierlicken Gruppe, Math. Ann. vol. 97 (1927) pp. 737-755.

64. L. Pontrjagin, Über stetige algebraische Körper, Ann. of Math. vol. 33 (1932) pp. 163-174.

65. - Topological groups, Princeton, 1939.

66. D. Raikov, To the theory of normed rings with involution, C. R. (Doklady) Acad. Sci. URSS. vol. 54 (1946) pp. 387-390.

67. C. Rickart, Banach algebras with an adioint operation, Ann. of Math. vol. 47 (1946) pp. 528-550.

68. - The singular elements of a Banach algebra, Duke Math. J. vol. 14 (1947) pp. 1063-1077.

69. I. Segal, The group algebra of a locally compact group, Trans. Amer. Math. Soc. vol. 61 (1947) pp. 69-105.

70. - Irreducible representations of operator algebras, Bull. Amer. Math. Soc. vol. 53 (1947) pp. 73-88.

71. - Postulates for general quantum mechanics, Ann. of Math. vol. 48 (1947) pp. 930-948.

72. I. Shafarevich, On the normalizability of topological fields, C. R. (Doklady) Acad. Sci. URSS. vol. 40 (1943) pp. 133-135.

73. G. Silov, On the extension of maximal ideals, C. R. (Doklady) Acad. Sci. URSS. vol. 29 (1940) pp. 83-84.

74. - On regular normed rings, Travaux de l'institut mathématique Stekloff, Moscow, 1947.

75. M. H. Stone, Applications of the theory of Boolean rings to general topology, 
Trans. Amer. Math. Soc. vol. 41 (1937) pp. 375-481.

76. O. Taussky, Analytical methods in hypercomplex systems, Compositio Math. vol. 3 (1936) pp. 399-407.

77. A. E. Taylor, The resolvent of a closed transformation, Bull. Amer. Math. Soc. vol. 44 (1938) pp. 70-74.

78. - Analysis in complex Banach spaces, Bull. Amer. Math. Soc. vol. 49 (1943) pp. 652-669.

79. O. Teichmüller, Diskret bewertete perfekte Körper mit unvollkommenem Restklassenkörper, Journal für Mathematik vol. 176 (1937) pp. 141-152.

80. A. Weil, L'intégration dans les groupes topologiques et ses applications, Actualites Scientifique et Industrielles, no. 869, Paris, 1938.

81. $\mathrm{K}$. Yosida, On the groups embedded in the metrical complete ring, Jap. J. Math. vol. 13 (1936) pp. 7-26.

82. - Normed rings and spectral theorems I-VI, Proc. Imp. Acad. Tokyo vols. 19 and 20.

83. D. Zelinsky, Topological characterization of fields with valuations, To appear in Bull Amer. Math. Soc.

University of Chicago 